QUARTERLY OF APPLIED MATHEMATICS

VOLUME LXVI, NUMBER 2

JUNE 2008, PAGES 281-302

S 0033-569X(08)01109-5

Article electronically published on February 7, 2008

\title{
SINGULAR SHOCK WAVES IN INTERACTIONS
}

\author{
BY \\ MARKO NEDELJKOV \\ Department of Mathematics and Informatics, University of Novi Sad, Trg D. Obradovića 4, 21000 \\ Novi Sad, Yugoslavia
}

\begin{abstract}
In a number of papers it has been shown that there exist one-dimensional systems such that they contain solutions with so-called overcompressive singular shock waves besides the usual elementary waves (shock and rarefaction waves as well as contact discontinuities).

One can see their definition for a general $2 \times 2$ system with fluxes linear in one of the dependent variables in Nedeljkov, Delta and singular delta locus for one dimensional systems of conservation laws, Math. Method Appl. Sci. 27 (2004), 931-955. This paper is devoted to examining their interactions with themselves and elementary waves. After a discussion of systems given in a general form, a complete analysis will be given for the ion-acoustic system given in Keyfitz and Kranzer, Spaces of weighted measures for conservation laws with singular shock solutions, J. Differ. Equations 118 (1995), no. 2, $420-451$.
\end{abstract}

1. Introduction. Consider the system

$$
\begin{aligned}
& \left(f_{2}(u)\right)_{t}+\left(f_{3}(u) v+f_{4}(u)\right)_{x}=0 \\
& \left(g_{1}(u) v+g_{2}(u)\right)_{t}+\left(g_{3}(u) v+g_{4}(u)\right)_{x}=0,
\end{aligned}
$$

where $f_{i}, g_{j}, i=2, \ldots, 4, j=1, \ldots, 4$ are polynomials with the maximal degree $m,(u, v)=$ $(u(x, t), v(x, t))$ are unknown functions with a physical range $\Omega,(x, t) \in \mathbb{R}_{+}^{2}:=\mathbb{R} \times \mathbb{R}_{+}$. We shall fix the following notation for the rest of the paper:

$$
f_{i}(y)=\sum_{k=0}^{m} a_{i, k} y^{k}, g_{j}(y)=\sum_{k=0}^{m} b_{j, k} y^{k}, i=2,3,4, j=1,2,3,4 .
$$

There are cases when there is no classical solution to the Riemann problem for the above system. Sometimes, there is a solution in the form of a delta or singular shock wave. In [8], one can see when a system in its evolution form (i.e. when $f_{2}=u, g_{1}=1$

Received June 10, 2006.

2000 Mathematics Subject Classification. Primary 35L65, 35L67.

Key words and phrases. conservation law systems, singular shock wave, interaction of singularities. The work is supported by Serbian Ministry of Science and Enviroment Protection, Grant No. 144016.

E-mail address: markonne@uns.ns.ac.yu, marko@im.ns.ac.yu 
and $\left.g_{2}=0\right)$ permits a solution in the shape of a singular shock wave. With the same type of reasoning and more effort, one can answer the same question in the case system (1.1).

The aim of this paper is to investigate the interaction of a singular shock wave with another wave. After a general statement about new initial data taken at the interaction point (of course, true for delta shock waves, too) in Section 3, we shall present a detailed investigation in the case of the system (the so-called ion-acoustic system)

$$
\begin{aligned}
u_{t}+\left(u^{2}-v\right)_{x} & =0 \\
v_{t}+\left(u^{3} / 3-u\right)_{x} & =0
\end{aligned}
$$

given in 6.

Definitions and concepts used here are similar to the ones used in [8], based on the notion of approximate solution. They are described in Section 2. Shortly, a solution to the above system is given by a net of smooth functions with equality substituted by a distributional limit.

A few interesting facts observed during the investigations of system (1.2) are raising a question about the possibilities in a general case. The observed facts are:

(1) The singular shock wave solution to a Riemann problem for (1.2) always has an increasing strength of the rate $\mathcal{O}(t), t \rightarrow \infty$. (The strength of the shock is a function that multiplies the delta function contained in a solution, $s(t)$ in (2.8).) After the interaction, the resulting singular shock wave is supported by a curve, not necessarily a straight line as before, and its strength can be an increasing, a constant, or a decreasing function with the respect to the time variable.

(2) When the resulting singular shock wave has a decreasing strength (this occurs during an interaction of a admissible singular shock wave with a rarefaction wave), after some time it can decompose into two shock waves. This is quite a new phenomenon.

The structure of this paper can be described in the following way.

In the second section we will introduce necessary notation and give basic notions based on the paper $[8]$.

In the third section, one can find a way how to continue a solution to the general case of system (1.1) after an interaction point (Theorem 3.5). The basic assumption is that the left-hand side of the first wave and the right-hand side of the second wave can be connected by a new singular shock wave. The conditions for such a possibility are formulated through the notion of second delta singular locus; see Definition 3.1 Explicit calculations for a geometric description of the locus are possible to some extent in the case of system (1.1), but we shall omit them completely.

The results given in these sections are used in subsequent sections devoted to special case (1.2), and that is the main part of the paper.

The first part of the fourth section is devoted to description of a situation which can occur after a singular shock and a shock wave interact. One can do the same for two singular shock waves, as one can at the end of this section.

The final, 5th section, contains the most interesting and important results about singular shock and rarefaction wave interaction. In that case, the decoupling of a singular 
shock into a pair of shock waves, already mentioned above, can occur. The analysis is performed when a singular shock wave is on the left-hand side of a rarefaction wave, but one can easily see that these results can be obtained using the same procedure when a singular shock is on the other side of a rarefaction wave.

We hope that the present paper gives some opportunity for extending the procedure in this paper for arbitrary initial data when a system possesses a singular or delta shock wave as a solution.

2. Notation. For a net of smooth functions, $U_{\varepsilon}: \mathbb{R}_{+}^{2} \rightarrow \Omega, \varepsilon \in(0,1)$ is distributionally zero, $U_{\varepsilon} \approx 0$, if $\iint U_{\varepsilon} \phi d x d t \rightarrow 0$ as $\varepsilon \rightarrow 0$ for every $\phi \in \mathcal{C}_{0}^{\infty}\left(\mathbb{R}_{+}^{2}\right)$. A maximal positive real $\alpha$ such that $\iint U_{\varepsilon} \phi d x d t=\mathcal{O}\left(\varepsilon^{\alpha}\right)$ as $\varepsilon \rightarrow 0$ is called the rate of convergence. Due to the linearity, $U_{\varepsilon} \approx V_{\varepsilon}$ if $U_{\varepsilon}-V_{\varepsilon} \approx 0$.

We say that a smooth function net $\left(u_{\varepsilon}, v_{\varepsilon}\right)$ is an approximate solution to (1.1) if

$$
\begin{aligned}
\iint\left(f_{2}\left(u_{\varepsilon}\right) \phi_{t}+\left(f_{3}\left(u_{\varepsilon}\right) v_{\varepsilon}+f_{4}\left(u_{\varepsilon}\right)\right) \phi_{x}\right) d x d t & \rightarrow 0 \\
\iint\left(\left(g_{1}\left(u_{\varepsilon}\right) v_{\varepsilon}+g_{2}\left(u_{\varepsilon}\right)\right) \phi_{t}+\left(g_{3}\left(u_{\varepsilon}\right) v_{\varepsilon}+g_{4}\left(u_{\varepsilon}\right)\right) \phi_{x}\right) d x d t & \rightarrow 0
\end{aligned}
$$

as $\varepsilon \rightarrow 0$ for every $\phi \in \mathcal{C}_{0}^{\infty}\left(\mathbb{R}_{+}^{2}\right)$. The above means

$$
\begin{aligned}
\left(f_{2}\left(u_{\varepsilon}\right)\right)_{t}+\left(f_{3}\left(u_{\varepsilon}\right) v_{\varepsilon}+f_{4}\left(u_{\varepsilon}\right)\right)_{x} & \approx 0 \\
\left(g_{1}\left(u_{\varepsilon}\right) v_{\varepsilon}+g_{2}\left(u_{\varepsilon}\right)\right)_{t}+\left(g_{3}\left(u_{\varepsilon}\right) v_{\varepsilon}+g_{4}\left(u_{\varepsilon}\right)\right)_{x} & \approx 0 .
\end{aligned}
$$

A net $G_{\varepsilon}$ is said to be of a bounded type if

$$
\sup _{(x, t) \in \mathbb{R} \times(0, T)}\left|G_{\varepsilon}(x, t)\right|=\mathcal{O}(1) \text { as } \varepsilon \rightarrow 0,
$$

for every $T>0$.

Before the definition of basic building blocks for a solution to the Riemann problem for (1.1), we give some useful functions.

Definition 2.1. (a) A net $G_{\varepsilon}$ is said to be a step function with value $\left(y_{0}, y_{1}\right)$ if it is of bounded type and

$$
G_{\varepsilon}(y)=\left\{\begin{array}{ll}
y_{0} & y<-\varepsilon \\
y_{1}, & y>\varepsilon
\end{array} .\right.
$$

Denote $[G]:=y_{1}-y_{0}$.

(b) A net $D_{\varepsilon}$ is said to be a split delta function ( $\mathrm{S} \delta$-function, for short) with value $\left(\alpha_{0}, \alpha_{1}\right)$ if $D_{\varepsilon}=\alpha_{0} D_{\varepsilon}^{-}+\alpha_{1} D_{\varepsilon}^{+}$, where $\alpha_{0}+\alpha_{1}=1$ and

$$
D_{\varepsilon} G_{\varepsilon} \approx\left(y_{0} \alpha_{0}+y_{1} \alpha_{1}\right) \delta
$$

for every step function $G_{\varepsilon}$ with value $\left(y_{0}, y_{1}\right)$.

(c) Let $m$ be an odd positive integer. A net $d_{\varepsilon}$ is said to be an $m^{\prime}$-singular delta function ( $m^{\prime}$ SD-function, for short) with value $\left(\beta_{0}, \beta_{1}\right)$ if $d_{\varepsilon}=\beta_{0} d_{\varepsilon}^{-}+\beta_{1} d_{\varepsilon}^{+}$, $\beta_{0}^{m-1}+\beta_{1}^{m-1}=1,\left(d_{\varepsilon}^{ \pm}\right)^{i} \approx 0, i \in\{1, \ldots, m-2, m\},\left(d_{\varepsilon}^{ \pm}\right)^{m-1} \approx \delta$, and

$$
d_{\varepsilon}^{m-1} G_{\varepsilon} \approx\left(y_{0} \beta_{0}^{m-1}+y_{1} \beta_{1}^{m-1}\right) \delta
$$

for every step function $G_{\varepsilon}$ with value $\left(y_{0}, y_{1}\right)$. 
(d) Let $m$ be an odd positive integer. A net $d_{\varepsilon}$ is said to be an $m$-singular delta function ( $m$ SD-function, for short) with value $\left(\beta_{0}, \beta_{1}\right)$ if $d_{\varepsilon}=\beta_{0} d_{\varepsilon}^{-}+\beta_{1} d_{\varepsilon}^{+}$, $\beta_{0}^{m}+\beta_{1}^{m}=1,\left(d_{\varepsilon}^{ \pm}\right)^{i} \approx 0, i \in\{1, \ldots, m-1\},\left(d_{\varepsilon}^{ \pm}\right)^{m} \approx \delta$, and

$$
d_{\varepsilon}^{m} G_{\varepsilon} \approx\left(y_{0} \beta_{0}^{m}+y_{1} \beta_{1}^{m}\right) \delta
$$

for every step function $G_{\varepsilon}$ with value $\left(y_{0}, y_{1}\right)$.

In this paper we shall assume the compatibility condition $D_{\varepsilon} d_{\varepsilon} \approx 0$, where $D_{\varepsilon}$ is an $\mathrm{S} \delta$-function and $d_{\varepsilon}$ is an $m \mathrm{SD}$ - or $m^{\prime} \mathrm{SD}$-function.

Suppose that the initial data are given by

$$
\left.u\right|_{t=T}=\left\{\begin{array}{ll}
u_{0}, & x<X \\
u_{1}, & x>X
\end{array},\left.\quad v\right|_{t=T}= \begin{cases}v_{0}, & x<X \\
v_{1}, & x>X .\end{cases}\right.
$$

According to our model, the initial data can be regularized to be

$$
\left.u\right|_{t=T}=G_{\varepsilon} \text { and }\left.v\right|_{t=T}=H_{\varepsilon},
$$

where $G_{\varepsilon}$ and $H_{\varepsilon}$ are step functions with values $\left(u_{0}, u_{1}\right)$ and $\left(v_{0}, v_{1}\right)$, respectively.

The classical waves in the net interpretation are given in the following way. (One can look in [2] or 7] for the definition of the classical waves.)

DeFinition 2.2. An approximate shock wave solution to (1.1) with the initial data (2.4) is

$$
\begin{aligned}
& u_{\varepsilon}(x, t)=G_{\varepsilon}(x-X-c(t-T)) \\
& v_{\varepsilon}(x, t)=H_{\varepsilon}(x-X-c(t-T)),
\end{aligned}
$$

where $c$ is the speed of the wave, and $G_{\varepsilon}$ and $H_{\varepsilon}$ are given by (2.5). The wave is admissible if it satisfies Lax conditions (with $\lambda_{2}>\lambda_{1}$ )

$$
\lambda_{1}\left(u_{0}, v_{0}\right)>c>\lambda_{1}\left(u_{1}, v_{1}\right) \text { or } \lambda_{2}\left(u_{0}, v_{0}\right)>c>\lambda_{2}\left(u_{1}, v_{1}\right),
$$

provided that the appropriate field is genuinely nonlinear.

An $i$-th approximate rarefaction wave solution to (1.1) with the initial data (2.4) is

$$
\left(u_{\varepsilon}, v_{\varepsilon}\right)(x, t)= \begin{cases}\left(u_{0}, v_{0}\right), & \frac{x-X}{t-T}<\lambda_{i}\left(u_{0}, v_{0}\right)-\varepsilon \\ \left(R_{i, u}, R_{i, v}\right)\left(\frac{x-X}{t-T}\right), & \lambda_{i}\left(u_{0}, v_{0}\right)+\varepsilon<\frac{x-X}{t-T}<\lambda_{i}\left(u_{1}, v_{1}\right)-\varepsilon \\ \left(u_{1}, v_{1}\right), & \frac{x-X}{t-T}>\lambda_{i}\left(u_{1}, v_{1}\right)+\varepsilon,\end{cases}
$$

where $u_{\varepsilon}$ and $v_{\varepsilon}$ are of the bounded type, while $\left(R_{i, u}, R_{i, v}\right)$ are self-similar non-constant solutions to (1.1) (non-constant parts of the classical $i$-th rarefaction wave solution to the system).

One can prove immediately that (2.6) is an approximate solution to the given system if and only if

$$
(u, v)(x, t)= \begin{cases}\left(u_{0}, v_{0}\right), & x-X<c(t-T) \\ \left(u_{1}, v_{1}\right), & x-X>c(t-T)\end{cases}
$$

satisfy the usual Rankine-Hugoniot conditions for the system. Also, one can see directly that (2.7) is an approximate solution, too.

There are no contact discontinuities used in this paper, but one can define its approximate version in the same way as we did for an approximate shock wave. 
One will see later that we do not require the rarefaction wave to be a smooth function, only a continuous one. Thus the above definition of an approximate rarefaction wave can be replaced by the rarefaction wave itself.

Now, we define the non-standard type of the wave used in the present paper.

DEFINITION 2.3. A singular shock wave (DSSW for short) is an approximate solution to (1.2) with the initial data (2.4) of the form

$$
\begin{aligned}
& u_{\varepsilon}(x, t)=G_{\varepsilon}((x-X)-c(t-T)) \\
& +\tilde{s}(t)\left(\alpha_{0} d_{\varepsilon}^{-}((x-X)-c(t-T))+\alpha_{1} d_{\varepsilon}^{+}((x-X)-c(t-T))\right) \\
& v_{\varepsilon}(x, t)=H_{\varepsilon}((x-X)-c(t-T)) \\
& +s(t)\left(\beta_{0} D_{\varepsilon}^{-}((x-X)-c(t-T))+\beta_{1} D_{\varepsilon}^{+}((x-X)-c(t-T))\right) \\
& +\tilde{\tilde{s}}(t)\left(\gamma_{0} d_{\varepsilon}^{-}((x-X)-c(t-T))+\gamma_{1} d_{\varepsilon}^{+}((x-X)-c(t-T))\right),
\end{aligned}
$$

where

(i) $c \in \mathbb{R}$ is the speed of the wave,

(ii) $s(t), \tilde{s}(t)$, and $\tilde{\tilde{s}}(t)$ are smooth functions for $t \geq 0$, and are equal to zero at $t=T$,

(iii) $G_{\varepsilon}$ and $H_{\varepsilon}$ are step functions with values $\left(u_{0}, u_{1}\right)$ and $\left(v_{0}, v_{1}\right)$, respectively,

(iv) $d_{\varepsilon}^{1}=\alpha_{0} d_{\varepsilon}^{-}+\beta_{1} d_{\varepsilon}^{+}$and $d_{\varepsilon}^{2}=\gamma_{0} d_{\varepsilon}^{-}+\gamma_{1} d_{\varepsilon}^{+}$are $m$ SD- or $m^{\prime}$ SD-functions, and

(v) $D_{\varepsilon}=\alpha_{0} D_{\varepsilon}^{-}+\alpha_{1} D_{\varepsilon}^{+}$is an $\mathrm{S} \delta$-function compatible with $d^{i}, i=1,2$.

The singular part of the wave is

$$
\left[\begin{array}{c}
\tilde{s}(t)\left(\alpha_{0} d_{\varepsilon}^{-}+\alpha_{1} d_{\varepsilon}^{+}\right) \\
s(t)\left(\beta_{0} D_{\varepsilon}^{-}+\beta_{1} D_{\varepsilon}^{+}\right)+\tilde{\tilde{s}}(t)\left(\gamma_{0} d_{\varepsilon}^{-}+\gamma_{1} d_{\varepsilon}^{+}\right)
\end{array}\right] .
$$

The wave is overcompressive if its speed is less or equal to the left-hand side characteristics, or greater or equal to the right-hand side characteristics, i.e.

$$
\lambda_{2}\left(u_{0}, v_{0}\right)>\lambda_{1}\left(u_{0}, v_{0}\right) \geq c \geq \lambda_{2}\left(u_{1}, v_{1}\right)>\lambda_{1}\left(u_{1}, v_{1}\right) .
$$

In solving the interaction problem below, the form of (2.8) will be changed in the following way. The values on the left- and/or the right-hand side of a DSSW can be non-constant functions, which would imply that the speed of the DSSW becomes nonconstant, $c=c(t)$. If a DSSW is involved in the interaction, then $s(T)$ will equal a strength of incoming DSSWs, not zero as in the case of initial data (2.4).

REMARK 2.4. (a) In [8] one can find special choices for $\mathrm{S} \delta$ - and $m \mathrm{SD}$ - or $m^{\prime} \mathrm{SD}$ functions. For example, $D_{\varepsilon}^{ \pm}$are given by the representatives

$$
D_{\varepsilon}^{ \pm}(y):=\frac{1}{\varepsilon} \phi\left(\frac{y-( \pm 2 \varepsilon)}{\varepsilon}\right)
$$

where $\phi \in \mathcal{C}_{0}^{\infty}$ is non-negative and even, supp $\phi \subset[-1,1] . m \mathrm{SD}$ - and $m^{\prime}$ SD-functions can be chosen in the same manner, with different shifts of their arguments and with $\phi^{1 / m}$ or $\phi^{1 /(m-1)}$ instead of $\phi$.

(b) The compatibility condition for an $\mathrm{S} \delta$-function $D$ and an $m \mathrm{SD}$ - or $m^{\prime} \mathrm{SD}$-function $d$ is automatically fulfilled if

$$
\operatorname{supp} d_{\varepsilon}^{+} \cap \operatorname{supp} D_{\varepsilon}^{+}=\operatorname{supp} d_{\varepsilon}^{-} \cap \operatorname{supp} D_{\varepsilon}^{-}=\emptyset .
$$

(c) The idea behind the above definition of products (2.1), (2.2), and (2.3) is the following. The starting point is that we know nothing about the infinitesimal values of 
the initial data (carried on by step functions $G_{\varepsilon}$ and $H_{\varepsilon}$ above) around zero, but only that any such unmeasurable influence stops at the points $\pm \varepsilon$. The above-mentioned definitions are made in order to obtain uniqueness of all products where step functions, $\mathrm{S} \delta$-, $m \mathrm{SD}-$, and $m^{\prime}$ SD-functions appear. With additional information for $G_{\varepsilon}$ and $H_{\varepsilon}$ around zero, one can choose $D_{\varepsilon}$ and $d_{\varepsilon}$ much more freely. For example, if $G_{\varepsilon}$ and $H_{\varepsilon}$ are monotone functions (which is a quite natural assumption), then relation (2.1) can be substituted by

$$
D_{\varepsilon} G_{\varepsilon} \approx \gamma \delta, \gamma \text { can be any real between } \min \left\{y_{0}, y_{1}\right\} \text { and } \max \left\{y_{0}, y_{1}\right\} \text {. }
$$

(d) The following result is proved in [6], Theorem 3. Suppose that a solution $\left(u_{\varepsilon}, v_{\varepsilon}\right)$ to a Dafermos-DiPerna viscosity approximation of (1.2),

$$
\begin{aligned}
u_{t}+\left(u^{2}-v\right)_{x} & =\varepsilon t u_{x x} \\
v_{t}+\left(u^{3} / 3-u\right)_{x} & =\varepsilon t v_{x x} \\
\left.(u, v)\right|_{t=0} & = \begin{cases}\left(u_{0}, v_{0}\right), & x<0 \\
\left(u_{1}, v_{1}\right), & x>0,\end{cases}
\end{aligned}
$$

is unbounded with respect to $\varepsilon$ and approaches a constant state as $\varepsilon \rightarrow 0$ except the line $x=c t$. Then $\left(u_{0}, v_{0}\right)$ and $\left(u_{1}, v_{1}\right)$ can be joined by an overcompressive DSSW.

That is a motivation to use overcompressiveness as an admissibility condition for DSSW. Note that the same condition is used for delta shock waves, too (see [5] or [10], for example).

(e) The DSSWs are much more peculiar than the delta shock waves. The latter can be viewed as elements of certain measure spaces (see [5] or [9], for example), which, when inserted into a system, solves it in an appropriate sense. A DSSW viewed as a net of smooth functions converges to weighted measure spaces defined in [6], but the obtained limits cannot be inserted back into the system. That is the reason why we use only nets of smooth functions for DSSWs, shock waves, and rarefaction waves.

Definition 2.5. The set of all points $\left(u_{1}, v_{1}\right) \in \Omega$ such that there exists a singular shock wave solution (called the corresponding DSSW) to Cauchy problem (1.1, 2.4) is called a delta singular locus. We shall write $\left(u_{1}, v_{1}\right) \in \operatorname{DSL}\left(u_{0}, v_{0}\right)$. If the corresponding DSSW is overcompressive, then it is called an overcompressive delta singular locus. We shall write $\left(u_{1}, v_{1}\right) \in \operatorname{DSL}^{*}\left(u_{0}, v_{0}\right)$.

In the sequel, the term "solution" will always denote the approximate one. Also, we shall drop the subscript $\varepsilon$ in the approximate solution. One has to keep in mind that any function depends on $\varepsilon$.

3. The new initial data. Suppose that system (1.1) posses a DSSW solution for some initial data. Assume one of the following.

(i) If an $m \mathrm{SD}$-function is contained in the above DSSW, then assume

$$
\operatorname{deg}\left(g_{1}\right)<m-1, \operatorname{deg}\left(g_{2}\right)<m, \operatorname{deg}\left(f_{2}\right)<m .
$$

(ii) If an $m^{\prime} \mathrm{SD}$-function is contained in the above DSSW, then assume

$$
\operatorname{deg}\left(g_{1}\right)<m-2, \operatorname{deg}\left(g_{2}\right)<m-1, \operatorname{deg}\left(f_{2}\right)<m-1 .
$$


Take the new initial data

$$
\left.u\right|_{t=T}=\left\{\begin{array}{ll}
u_{0}, & x<X \\
u_{1}, & x>X
\end{array},\left.v\right|_{t=T}=\left\{\begin{array}{ll}
v_{0}, & x<X \\
v_{1}, & x>X
\end{array}+\zeta \delta_{(X, T)},\right.\right.
$$

for system (1.1), where $\zeta$ is a non-zero real.

Definition 3.1. The set of all points $\left(u_{1}, v_{1}\right) \in \Omega$ such that there exists a DSSW solution (called the corresponding DSSW) to Cauchy problem (1.1, 3.3) for some $\zeta$ is called a second delta singular locus of initial strength $\zeta$ for $\left(u_{0}, v_{0}\right)$. We shall write $\left(u_{1}, v_{1}\right) \in$ $\operatorname{SDSL}_{\zeta}\left(u_{0}, v_{0}\right)$. If the corresponding DSSW is overcompressive, then it is called an overcompressive second delta singular locus, and we shall write $\left(u_{1}, v_{1}\right) \in \operatorname{SDSL}_{\zeta}^{*}\left(u_{0}, v_{0}\right)$.

Before the main theorem, let us give a useful lemma.

Lemma 3.2. Suppose that $\left(u_{1}, v_{1}\right) \in \operatorname{DSL}\left(u_{0}, v_{0}\right)$. Then $\left(u_{1}, v_{1}\right) \in \operatorname{SDSL}_{\zeta}\left(u_{0}, v_{0}\right)$ if $\zeta>0$.

If the corresponding DSSW contains an $m$ SD-function, and $m$ is an odd number, then the statement holds true for every real $\zeta$.

Additionally, $\beta_{i}, i=1,2$, from Definition 2.3 for the corresponding DSSW do not depend on $\zeta$.

Proof. We shall give the proof for a DSSW containing an $m$ SD-function (2.8). The other case can be proved in the same way.

Inserting functions $u$ and $v$ from (2.8) into system (1.1) with initial data (3.3) and taking into account relations (3.1) or (3.2), one gets

$$
\begin{aligned}
f_{2}(u) \approx & f_{2}(G) \\
g_{1}(u) \approx & g_{1}(G) \\
g_{2}(u) \approx & g_{2}(G) \\
f_{3}(u) \approx & f_{3}(G)+\tilde{s}(t)^{m-1}\left(u_{1} \alpha_{0}^{m-1}\left(d^{-}\right)^{m-1}+u_{0} \alpha_{1}^{m-1}\left(d^{+}\right)^{m-1}\right) m a_{3, m} \\
& +\tilde{s}(t)^{m}\left(\alpha_{0}^{m}\left(d^{-}\right)^{m}+\alpha_{1}^{m}\left(d^{+}\right)^{m}\right) a_{3, m} \\
& +\tilde{s}(t)^{m-1}\left(\alpha_{0}^{m-1}\left(d^{-}\right)^{m-1}+\alpha_{1}^{m-1}\left(d^{+}\right)^{m-1}\right) a_{3, m-1} \\
f_{4}(u) \approx & f_{4}(G)+\tilde{s}(t)^{m-1}\left(u_{1} \alpha_{0}^{m-1}\left(d^{-}\right)^{m-1}+u_{0} \alpha_{1}^{m-1}\left(d^{+}\right)^{m-1}\right) m a_{4, m} \\
& +\tilde{s}(t)^{m}\left(\alpha_{0}^{m}\left(d^{-}\right)^{m}+\alpha_{1}^{m}\left(d^{+}\right)^{m}\right) a_{4, m} \\
& +\tilde{s}(t)^{m-1}\left(\alpha_{0}^{m-1}\left(d^{-}\right)^{m-1}+\alpha_{1}^{m-1}\left(d^{+}\right)^{m-1}\right) a_{4, m-1} \\
g_{3}(u) \approx & g_{3}(G)+\tilde{s}(t)^{m-1}\left(u_{1} \alpha_{0}^{m-1}\left(d^{-}\right)^{m-1}+u_{0} \alpha_{1}^{m-1}\left(d^{+}\right)^{m-1}\right) m b_{3, m} \\
& +\tilde{s}(t)^{m}\left(\alpha_{0}^{m}\left(d^{-}\right)^{m}+\alpha_{1}^{m}\left(d^{+}\right)^{m}\right) b_{3, m} \\
& +\tilde{s}(t)^{m-1}\left(\alpha_{0}^{m-1}\left(d^{-}\right)^{m-1}+\alpha_{1}^{m-1}\left(d^{+}\right)^{m-1}\right) b_{3, m-1} \\
g_{4}(u) \approx & g_{4}(G)+\tilde{s}(t)^{m-1}\left(u_{1} \alpha_{0}^{m-1}\left(d^{-}\right)^{m-1}+u_{0} \alpha_{1}^{m-1}\left(d^{+}\right)^{m-1}\right) m b_{4, m} \\
& +\tilde{s}(t)^{m}\left(\alpha_{0}^{m}\left(d^{-}\right)^{m}+\alpha_{1}^{m}\left(d^{+}\right)^{m}\right) b_{4, m} \\
& +\tilde{s}(t)^{m-1}\left(\alpha_{0}^{m-1}\left(d^{-}\right)^{m-1}+\alpha_{1}^{m-1}\left(d^{+}\right)^{m-1}\right) b_{4, m-1} .
\end{aligned}
$$

There are two possible cases. Either $\tilde{\tilde{s}} \not \equiv 0$ and $a_{3, m}=b_{3, m}=0$ (i.e. $\operatorname{deg}\left(f_{3}\right) \leq m-1$ and $\left.\operatorname{deg}\left(g_{3}\right) \leq m-1\right)$, or $\tilde{\tilde{s}} \equiv 0$. In both the cases, the procedure that follows is the 
same, so take $\tilde{\tilde{s}} \not \equiv 0$ and $a_{3, m}=b_{3, m}=0$. From the first equation of (1.1), one gets

$$
\begin{aligned}
& \left(f_{2}(u)\right)_{t}+\left(f_{3}(u) v+f_{4}(u)\right)_{x} \\
\approx & -c\left(\left[f_{2}(G)\right]+\left[f_{3}(G) H+f_{4}(G)\right]\right) \delta \\
& +\tilde{s}(t)^{m-1} \tilde{\tilde{s}}(t)\left(u_{1} \alpha_{0}^{m-1} \gamma_{0}+u_{0} \alpha_{1}^{m-1} \gamma_{1}\right) m a_{3, m-1} \delta^{\prime} \\
& +\left(f_{3}\left(u_{0}\right) \beta_{0}+f_{3}\left(u_{1}\right) \beta_{1}\right) \delta^{\prime}+\tilde{s}(t)^{m} a_{4, m} \delta^{\prime} \approx 0 .
\end{aligned}
$$

One immediately gets the speed of the DSSW,

$$
c=\frac{\left[f_{3}(G) H+f_{4}(G)\right]}{\left[f_{2}(G)\right]},
$$

and the relations

$$
\kappa_{1} s(t)=\tilde{s}(t)^{m-1} \tilde{\tilde{s}}(t) \text { and } \kappa_{2} s(t)=\tilde{s}(t)^{m},
$$

for some reals $\kappa_{1}$ and $\kappa_{2}$. Finally, one gets

$$
\kappa_{1}\left(u_{1} \alpha_{0}^{m-1} \gamma_{0}+u_{0} \alpha_{1}^{m-1} \gamma_{1}\right) m a_{3, m-1}+f_{3}\left(u_{0}\right) \beta_{0}+f_{3}\left(u_{1}\right) \beta_{1}+\kappa_{2} a_{4, m}=0 .
$$

Inserting all of these relations into the second equation, one gets

$$
\begin{aligned}
& \left.\left(g_{1}(u) v+g_{2}(u)\right)_{t}+\left(g_{3}(u) v+g_{4} u\right)\right)_{x} \\
\approx & \left(-c\left[g_{1}(G) H+g_{2}(G)\right]+\left[g_{3}(G) H+g_{4}(G)\right]+s^{\prime}(t)\left(g_{1}\left(u_{0}\right) \beta_{0}+g_{1}\left(u_{1}\right) \beta_{1}\right)\right) \delta \\
& +s(t)\left(g_{1}\left(u_{0}\right) \beta_{0}+g_{1}\left(u_{1}\right) \beta_{1}+g_{3}\left(u_{0}\right) \beta_{0}+g_{3}\left(u_{1}\right) \beta_{1}\right. \\
& \left.+\kappa_{1}\left(u_{1} \alpha_{0}^{m-1} \gamma_{0}+u_{0} \alpha_{1}^{m-1} \gamma_{1}\right) m b_{3, m-1}+\kappa_{2} b_{4, m}\right) \delta^{\prime} \approx 0 .
\end{aligned}
$$

The function $s$ must be a linear one, say $s^{\prime}(t)=\sigma$, and the above functional equation gives the last two equations in $\mathbb{R}$,

$$
-c\left[g_{1}(G) H+g_{2}(G)\right]+\left[g_{3}(G) H+g_{4}(G)\right]+\sigma\left(g_{1}\left(u_{0}\right) \beta_{0}+g_{1}\left(u_{1}\right) \beta_{1}\right)=0
$$

and

$$
\begin{aligned}
& -c\left(\left(g_{1}\left(u_{0}\right)+g_{3}\left(u_{0}\right) \beta_{0}+\left(g_{1}\left(u_{1}\right)+g_{3}\left(u_{1}\right) \beta_{1}\right)\right)\right) \\
& +\kappa_{1}\left(u_{1} \alpha_{0}^{m-1} \gamma_{0}+u_{0} \alpha_{1}^{m-1} \gamma_{1}\right) m b_{3, m-1}+\kappa_{2} b_{4, m}=0 .
\end{aligned}
$$

In the above equations, the only important fact about $s$ is its derivative. Thus one can safely put $s(t)=\sigma t+\zeta$, and if the above system (3.4 3.6) has a solution, then $\left(u_{1}, v_{1}\right) \in \operatorname{SDSL}_{\zeta}\left(u_{0}, v_{0}\right)$, provided that $\tilde{s}$ and $\tilde{\tilde{s}}$ can be recovered. Using the fact that $\left(u_{1}, v_{1}\right) \in \operatorname{DSL}\left(u_{0}, v_{0}\right)$, that is certainly the case when $\zeta>0$. If $m$ is an odd number, then $\tilde{s}=s(t)^{1 / m}$ and $\tilde{\tilde{s}}=\tilde{s}$ are always determined.

The second part of the assertion, that $\beta_{i}, i=1,2$ are independent of $\zeta$, is obvious from the above.

REMARK 3.3. From the proof of the lemma one can see that it is actually possible for $\zeta$ to take some negative values, i.e. it is enough that $\zeta \geq-s(T)$, where $T$ is a time of interaction when new initial data are given.

The following assertion is crucial for the construction of a weak solution (a solution in an associated sense) to (1.1) after an interaction: At an interaction point of a DSSW and some other wave, one can consider the new initial value problem that contains a delta function. 
Suppose that the initial data are given by

$$
u(x, 0)=\left\{\begin{array}{ll}
u_{0}, & x<a \\
u_{1}, & a<x<b \\
u_{2}, & x>b
\end{array} \text { and } v(x, 0)= \begin{cases}v_{0}, & x<a \\
v_{1}, & a<x<b \\
v_{2}, & x>b\end{cases}\right.
$$

such that there exist a DSSW starting from the point $x=a$ and a shock wave (or another DSSW) starting from the point $x=b, a<b$. They can interact if $c_{1}>c_{2}$, where $c_{i}$ is the speed of the $i$-th wave, $i=1,2$. For simplicity we shall assume that $b=0$.

Let $(X, T)$ be the interaction point of the overcompressive DSSW starting at the point $x=a$ :

$$
\begin{aligned}
u^{1}(x, t)= & G^{1}\left(x-c_{1} t-a\right)+\tilde{s}^{1}(t)\left(\alpha_{0}^{1} d^{-}\left(x-c_{1} t-a\right)+\alpha_{1}^{1} d^{+}\left(x-c_{1} t-a\right)\right) \\
v^{1}(x, t)= & H^{1}\left(x-c_{1} t-a\right)+s^{1}(t)\left(\beta_{0}^{1} D^{-}\left(x-c_{1} t-a\right)+\beta_{1}^{1} D^{+}\left(x-c_{1} t-a\right)\right) \\
& +\tilde{s}^{1}(t)\left(\gamma_{0}^{1} d^{-}\left(x-c_{1} t-a\right)+\gamma_{1}^{1} d^{+}\left(x-c_{1} t-a\right)\right),
\end{aligned}
$$

and the admissible (singular) shock wave

$$
\begin{aligned}
u^{2}(x, t)= & G^{2}\left(x-c_{2} t\right)+\tilde{s}^{2}(t)\left(\alpha_{0}^{2} d^{-}\left(x-c_{2} t\right)+\alpha_{1}^{2} d^{+}\left(x-c_{2} t\right)\right) \\
v^{2}(x, t)= & H^{2}\left(x-c_{2} t\right)+s^{2}(t)\left(\beta_{0}^{2} D^{-}\left(x-c_{2} t\right)+\beta_{1}^{2} D^{+}\left(x-c_{2} t\right)\right) \\
& +\tilde{s}^{2}(t)\left(\gamma_{0}^{2} d^{-}\left(x-c_{2} t-a\right)+\gamma_{1}^{2} d^{+}\left(x-c_{2} t-a\right)\right),
\end{aligned}
$$

where $G^{1}, G^{2}, H^{1}$, and $H^{2}$ are the generalized step functions with values $\left(u_{0}, u_{1}\right),\left(u_{1}, u_{2}\right)$, $\left(v_{0}, v_{1}\right)$ and $\left(v_{1}, v_{2}\right)$, respectively. Also, $\left(\alpha_{0}^{i}\right)^{m_{1}}+\left(\alpha_{1}^{i}\right)^{m_{1}}=\left(\gamma_{0}^{i}\right)^{m_{1}}+\left(\gamma_{1}^{i}\right)^{m_{1}}=\beta_{0}^{i}+\beta_{1}^{i}=1$, $i=1,2$. Here, $m_{1}=m$ if the singular part of the DSSW is an $m$ SD-function and $m_{1}=m-1$ in the case of an $m^{\prime}$ SD-function. If the second wave is a shock wave, then one can put $s^{2} \equiv \tilde{s}^{2} \equiv \tilde{\tilde{s}}^{2} \equiv 0$.

The speed of a DSSW (as well as for a shock wave) can be found using the first equation in (1.1) because of assumption (3.1) or (3.2). For example, in the case of the first DSSW (3.8), we have

$$
\begin{aligned}
& \left(f_{2}(u)\right)_{t}+\left(f_{3}(u) v+f_{4}(u)\right)_{x} \approx\left(f_{2}(G)\right)_{t}+\left(f_{3}(G) H+f_{4}(G)\right)_{x}+\left(\text { const } s^{1}(t) \delta\right)_{x} \\
\approx & \left(-c_{1}\left[f_{2}(G)\right]+\left[f_{3}(G) H+f_{4}(G)\right]\right) \delta+\text { const } s^{1}(t) \delta^{\prime} \approx 0,
\end{aligned}
$$

where the term const $s^{1}(t)$ is determined, but we shall not write the exact value since it is not needed for the assertion. The missing argument in the above expression is $x-c_{1} t-a$.

Let $\Gamma_{1}=\left\{x=c_{1} t+a\right\}$ and $\Gamma_{2}=\left\{x=c_{2} t\right\}$. Then $[\cdot]_{\Gamma_{i}}$ denotes the jump at the curve $\Gamma_{i}, i=1,2$. Thus, one can see that the speed of that DSSW has the same value as in the case of the shock wave,

$$
c_{1}=\frac{\left[f_{3}(G) H+f_{4}(G)\right]_{\Gamma_{1}}}{\left[f_{2}(G)\right]_{\Gamma_{1}}} .
$$

Also,

$$
c_{2}=\frac{\left[f_{3}(G) H+f_{4}(G)\right]_{\Gamma_{2}}}{\left[f_{2}(G)\right]_{\Gamma_{2}}} .
$$


Finally, one can see that the waves given by (3.8) and (3.9) will interact at the point $(X, T)$ if $a<0$ and $c_{1}>c_{2}$, where

$$
\begin{aligned}
T & =\frac{a\left[f_{2}(G)\right]_{\Gamma_{1}}\left[f_{2}(G)\right]_{\Gamma_{2}}}{\left[f_{3}(G) H+f_{4}(G)\right]_{\Gamma_{2}}\left[f_{2}(G)\right]_{\Gamma_{1}}-\left[f_{3}(G) H+f_{4}(G)\right]_{\Gamma_{1}}\left[f_{2}(G)\right]_{\Gamma_{2}}} \\
X & =\frac{a\left[f_{3}(G) H+f_{4}(G)\right]_{\Gamma_{2}}\left[f_{2}(G)\right]_{\Gamma_{1}}}{\left[f_{3}(G) H+f_{4}(G)\right]_{\Gamma_{2}}\left[f_{2}(G)\right]_{\Gamma_{1}}-\left[f_{3}(G) H+f_{4}(G)\right]_{\Gamma_{1}}\left[f_{2}(G)\right]_{\Gamma_{2}}} .
\end{aligned}
$$

Denote by $(\tilde{u}(x, t), \tilde{v}(x, t))$ a solution before the interaction time $t=T$ consisting of waves (3.8, 3.9).

REMARK 3.4. In the case of system (1.2) one can easily calculate speeds of the above shocks and coordinates of the interaction point. The speeds of DSSW and the entropy shock wave are

$$
c_{1}=\frac{u_{1}^{2}-v_{1}-u_{0}^{2}+v_{0}}{u_{1}-u_{0}} \text { and } c_{2}=\frac{u_{2}^{2}-v_{2}-u_{1}^{2}+v_{1}}{u_{2}-u_{1}} .
$$

If $c_{1}>c_{2}$, then one gets

$$
X=\frac{-a c_{2}}{c_{2}-c_{1}} \text { and } T=\frac{a}{c_{2}-c_{1}}
$$

for the interaction point $(X, T)$.

Theorem 3.5. Let system (1.1) be given. Suppose that $\left(u_{2}, v_{2}\right) \in \operatorname{SDSL}_{\zeta}\left(u_{0}, v_{0}\right), \zeta=$ $\left(\zeta_{1}+\zeta_{2}\right) /\left(g_{1}\left(u_{0}\right) \beta_{0}+g_{1}\left(u_{1}\right) \beta_{1}\right)$, where the constants $\zeta_{i}, i=1,2$, are defined by

$$
\begin{aligned}
& g_{1}\left(u^{1}\right) v^{1}+\left.g_{2}\left(u^{1}\right)\right|_{(t=T)} \approx \zeta_{1} \delta_{(X, T)} \\
& g_{1}\left(u^{2}\right) v^{2}+\left.g_{2}\left(u^{2}\right)\right|_{(t=T)} \approx \zeta_{2} \delta_{(X, T)} .
\end{aligned}
$$

The corresponding DSSW, $(\hat{u}, \hat{v})(x, t)$, is given by

$$
\begin{aligned}
\hat{u}(x, t)= & G(x-X-c(t-T)) \\
& +\tilde{s}(t)\left(\alpha_{0} d^{-}(x-X-c(t-T))+\alpha_{1} d^{+}(x-X-c(t-T))\right) \\
\hat{v}(x, t)= & H(x-X-c(t-T)) \\
& +s(t)\left(\beta_{0} D^{-}(x-X-c(t-T))+\beta_{1} D^{+}(x-X-c(t-T))\right) \\
& +\tilde{\tilde{s}}(t)\left(\gamma_{0} d^{-}(x-X-c(t-T))+\gamma_{1} d^{+}(x-X-c(t-T))\right)
\end{aligned}
$$

for $t>T$. By Lemma 3.2. $\beta_{0}$ and $\beta_{1}$ are determined independently on $\zeta$, so the definition of DSSW makes sense.

Then there exists a solution to (1.1 3.7) in the association sense such that it equals $(\tilde{u}, \tilde{v})(x, t)$ for $t<T-\varepsilon$, and it equals $(\hat{u}, \hat{v})(x, t)$ for $t>T+\varepsilon$.

Proof. Take a constant $t_{0}$ such that the singular parts of the waves $\left(u^{1}(x, t), v^{1}(x, t)\right)$ and $\left(u^{2}(x, t), v^{2}(x, t)\right)$ have disjoint supports (i.e. $c_{1} t-a-c_{2} t>4 \varepsilon$, for $t<T-t_{0} \varepsilon$, if one uses the construction of the $\mathrm{S} \delta, m \mathrm{SD}$ and $m^{\prime} \mathrm{SD}$-functions defined above). 
Let us denote

$$
\begin{aligned}
& \Delta_{\varepsilon}=\left\{(x, t):|x-X| \leq t_{0} \varepsilon+\varepsilon,|t-T| \leq t_{0} \varepsilon+\varepsilon\right\}, \\
& \tilde{\Delta}_{\varepsilon}=\left\{(x, t):|x-X| \leq t_{0} \varepsilon,|t-T| \leq t_{0} \varepsilon\right\}, \\
& A_{\varepsilon}=\left\{(x, t):|x-X| \leq t_{0} \varepsilon+\varepsilon, t=T-t_{0} \varepsilon-\varepsilon\right\}, \\
& B_{\varepsilon}=\left\{(x, t): x=X+t_{0} \varepsilon+\varepsilon,|t-T| \leq t_{0} \varepsilon+\varepsilon\right\}, \\
& C_{\varepsilon}=\left\{(x, t):|x-X| \leq t_{0} \varepsilon+\varepsilon, t=T+t_{0} \varepsilon+\varepsilon\right\}, \\
& D_{\varepsilon}=\left\{(x, t): x=X-t_{0} \varepsilon-\varepsilon,|t-T| \leq t_{0} \varepsilon+\varepsilon\right\} .
\end{aligned}
$$

Define a cut-off function $\xi_{\varepsilon}(x, t)$ that equals zero for $(x, t) \in \Delta_{\varepsilon}$ and 1 for $(x, t) \in$ $\mathbb{R}_{+}^{2} \backslash \tilde{\Delta}_{\varepsilon}$. Let

We shall prove that

$$
\left(u_{\text {temp }}, v_{\text {temp }}\right)(x, t)= \begin{cases}(\tilde{u}(x, t), \tilde{v}(x, t)), & t<T \\ (\hat{u}(x, t), \hat{v}(x, t)), & t>T .\end{cases}
$$

$$
u(x, t)=u_{\text {temp }}(x, t) \xi_{\varepsilon}(x, t) \text { and } v(x, t)=v_{\text {temp }}(x, t) \xi_{\varepsilon}(x, t), x \in \mathbb{R}, t \geq 0
$$

are approximate solutions to (1.1).

Denote

$$
\mathbf{F}(u, v)=\left[\begin{array}{c}
f_{2}(u) \\
g_{1}(u) v+g_{2}(u)
\end{array}\right] \text { and } \mathbf{G}(u, v)=\left[\begin{array}{l}
f_{3}(u) v+f_{4}(u) \\
g_{3}(u) v+g_{4}(u)
\end{array}\right] .
$$

We have

$$
\begin{aligned}
& \iint_{\mathbb{R}_{+}^{2}} \mathbf{F}(u, v) \Psi_{t}+\mathbf{G}(u, v) \Psi_{x} d x d t \\
= & \iint_{\tilde{\Delta}_{\varepsilon}} \mathbf{F}(u, v) \Psi_{t}+\mathbf{G}(u, v) \Psi_{x} d x d t \\
= & \iint_{\mathbb{R}_{+}^{2} \backslash \tilde{\Delta}_{\varepsilon}} \mathbf{F}(u, v) \Psi_{t}+\mathbf{G}(u, v) \Psi_{x} d x d t
\end{aligned}
$$

for every test function $\Psi=\left[\begin{array}{c}\psi_{1} \\ \psi_{2}\end{array}\right] \in \mathcal{C}_{0}^{\infty}\left(\mathbb{R}_{+}^{2}\right)$.

The measure of the set $\tilde{\Delta}_{\varepsilon}$ is $\mathcal{O}\left(\varepsilon^{2}\right)$ as $\varepsilon \rightarrow 0$, while

$$
\left\|\mathbf{F}(u, v) \Psi_{t}+\mathbf{G}(u, v) \Psi_{x}\right\|_{L^{\infty}\left(\mathbb{R}_{+}^{2}\right)} \leq \text { const } \varepsilon^{-1+1 / m}
$$

due to the assumptions in Definition 2.1. Thus,

$$
\iint_{\tilde{\Delta}_{\varepsilon}} \mathbf{F}(u, v) \Psi_{t}+\mathbf{G}(u, v) \Psi_{x} d x d t \sim \varepsilon^{1-1 / m} \rightarrow 0 \text { as } \varepsilon \rightarrow 0 .
$$

Using the divergence theorem for the second integral, one gets

$$
\begin{aligned}
& \iint_{\mathbb{R}_{+}^{2} \backslash \tilde{\Delta}_{\varepsilon}} \mathbf{F}(u, v) \Psi_{t}+\mathbf{G}(u, v) \Psi_{x} d x d t \\
= & \int_{\partial \tilde{\Delta}_{\varepsilon}} \mathbf{F}(u, v) \Psi \nu_{t}+\int \mathbf{G}(u, v) \Psi \nu_{x} d s \\
& -\iint_{\mathbb{R}_{+}^{2} \backslash \tilde{\Delta}_{\varepsilon}} \mathbf{F}(u, v)_{t} \Psi+\mathbf{G}(u, v)_{x} \Psi d x d t .
\end{aligned}
$$


The last integral in the above expression tends to zero as $\varepsilon \rightarrow 0$ since $(u, v)$ solves (1.1) in $\mathbb{R}_{+}^{2} \backslash \tilde{\Delta}_{\varepsilon}$ due to the construction. For the other one we have

$$
\begin{aligned}
& \int_{\partial \tilde{\Delta}_{\varepsilon}} \mathbf{F}(u, v) \Psi \nu_{t}+\int \mathbf{G}(u, v) \Psi \nu_{x} d s \\
= & \int_{A_{\varepsilon}} \mathbf{F}(u, v) \Psi d x-\int_{C_{\varepsilon}} \mathbf{F}(u, v) \Psi d x+\int_{D_{\varepsilon}} \mathbf{G}(u, v) \Psi d t-\int_{B_{\varepsilon}} \mathbf{G}(u, v) \Psi d t .
\end{aligned}
$$

Functions $u$ and $v$ are $L^{\infty}$-bounded uniformly in $\varepsilon$ on the sides $B_{\varepsilon}$ and $D_{\varepsilon}$. Since their lengths are $\mathcal{O}(\varepsilon)$, integrals over them tend to zero as $\varepsilon \rightarrow 0$.

Using the fact that $f_{2}(d) \approx 0$, one gets

$$
\left.\lim _{\varepsilon \rightarrow 0} F(\tilde{u}, \tilde{v})\right|_{t=T}=\left[\begin{array}{c}
0 \\
\left(\zeta_{1}+\zeta_{2}\right) \delta_{(X, T)}
\end{array}\right],
$$

as well as the construction of $\mathrm{S} \delta$ - and $m^{\prime} \mathrm{SD}$ (or $m \mathrm{SD}$ )-functions, one gets

$$
\lim _{\varepsilon \rightarrow 0} \int_{A_{\varepsilon}} \mathbf{F}(u, v) d x=\left[\begin{array}{c}
0 \\
\zeta_{1}+\zeta_{2}
\end{array}\right] \cdot \Psi(X, T) .
$$

Thus

$$
\lim _{\varepsilon \rightarrow 0} \int_{C_{\varepsilon}} \mathbf{F}(u, v) d x=-\left[\begin{array}{c}
0 \\
\zeta_{1}+\zeta_{2}
\end{array}\right] \cdot \Psi(X, T)
$$

has to be true. This implies $\left.f_{2}(\hat{u})\right|_{(X, T)} \approx 0$ and

$$
g_{1}(\hat{u}) \hat{v}+\left.g_{2}(\hat{u})\right|_{(X, T)} \approx\left(\zeta_{1}+\zeta_{2}\right) \delta_{(X, T)} .
$$

Due to conditions (3.1) or (3.2), one immediately gets $\left.f_{2}(\hat{u})\right|_{(X, T)} \approx 0$. Put $\zeta=$ $\left(\zeta_{1}+\zeta_{2}\right) /\left(g_{1}\left(u_{0}\right) \beta_{0}+g_{1}\left(u_{1}\right) \beta_{1}\right)$. Then

$$
g_{1}(\hat{u}) \hat{v}+\left.g_{2}(\hat{u})\right|_{t=T} \approx \hat{G} \hat{H}+\hat{G}+s(T)\left(g_{1}\left(u_{0}\right) \beta_{0}+g_{1}\left(u_{1}\right) \beta_{1}\right) \delta(X),
$$

and after another restriction on the point $x=X$,

$$
g_{1}(\hat{u}) \hat{v}+\left.g_{2}(\hat{u})\right|_{(X, T)} \approx\left(\zeta_{1}+\zeta_{2}\right) \delta_{(X, T)} .
$$

That concludes the proof.

REMARK 3.6. The distributional limit of the result of the interaction is given by

$$
\begin{aligned}
& u(x, t)=\left\{\begin{array}{l}
u_{0}, x<c_{1} t-a, t<t \\
u_{1}, c_{1} t-a<x<c_{2} t, t<T \\
u_{2}, x>c_{2} t, t<T \\
u_{0}, x<c t+X, t>T \\
u_{2}, x>c t+X, t>T
\end{array}\right. \\
& v(x, t)=\left\{\begin{array}{l}
v_{0}, x<c_{1} t-a, t<t \\
v_{1}, c_{1} t-a<x<c_{2} t, t<T \\
v_{2}, x>c_{2} t, t<T \\
v_{0}, x<c t+X, t>T \\
v_{2}, x>c t+X, t>T
\end{array}\right\}+s_{1}(t) \delta_{S_{1}}+s_{2}(t) \delta_{S_{2}}+s(t) \delta_{S},
\end{aligned}
$$


where $S_{1}=\left\{(x, t): x=c_{1} t+a, t \in[0, T]\right\}, S_{2}=\left\{(x, t): x=c_{2} t, t \in[0, T]\right\}$, and $S=\{(x, t): x-X=c(t-T), t \in[T, \infty)\}$. If the second wave (3.9) is a shock wave, then $s_{2} \equiv 0$.

The above solution is continuous in $t$ with values in $\mathcal{D}^{\prime}(\mathbb{R})$. This fact can be used in the approach similar to [3] and [4, where the variable $t$ is treated separately, i.e. when system (1.1) is considered to be in evolution form.

The theorem shows that, after an interaction of a DSSW with some shock or another DSSW, the problem reduces to solving system (1.1) with the new initial data (3.3).

REMARK 3.7. (i) The solution to the interaction problem from Theorem 3.5 is always associated with a lower association rate than the solution of the original Riemann problem. For specific systems it may be possible to make more sophisticated constructions in order to improve the rate.

(ii) It appears that $d^{ \pm}$are unavoidable correction factors, even though their distributional limit equals zero. The conditions (3.1) and (3.2) ensure that the new initial data at the intersection point do not depend on $m \mathrm{SD}$ - or $m^{\prime} \mathrm{SD}$-functions in the solution. We have used them because the real nature of $m \mathrm{SD}$ - and $m^{\prime} \mathrm{SD}$-functions is not so clear yet.

The above theorem will be used in the rest of the paper for the investigation of interactions between singular shock waves and other types of waves in the special case of system (1.2).

4. Applications. Consider now system (1.2), which is a special case of (1.1). The authors of [6] defined and proved existence of DSSW solutions for some Riemann problems of this system. In the present paper, we will investigate interactions of such solutions with the other solutions to the Riemann problem for (1.2). In order to familiarize the reader with the presented results, let us make some basic remarks about such solutions.

For given Riemann data $\left(u_{0}, v_{0}\right),\left(v_{1}, v_{1}\right)$, there are three basic solution types:

(a) Shock waves:

$$
u(x, t)=\left\{\begin{array}{l}
u_{0}, x<c t \\
u_{1}, x>c t
\end{array} \quad v(x, t)=\left\{\begin{array}{l}
v_{0}, x<c t \\
v_{1}, x>c t
\end{array}\right.\right.
$$

where $c=\left[u^{2}-v\right] /[u]$ and $\left(u_{1}, v_{1}\right)$ lies in an admissible part of the Hugoniot locus of the point $\left(u_{0}, v_{0}\right)$.

(b) Centered rarefaction waves:

$$
\begin{aligned}
& u(x, t)=\left\{\begin{array}{c}
u_{0}, x<\left(u_{0}-1\right) t \\
x / t+1,\left(u_{0}-1\right) t \leq x \leq\left(u_{1}-1\right) t \\
u_{1}, x>\left(u_{1}-1\right) t
\end{array}\right. \\
& v(x, t)=\left\{\begin{array}{c}
v_{0}, x<\left(u_{0}-1\right) t \\
(x / t)^{2} / 2+2 x / t+C_{1},\left(u_{0}-1\right) t \leq x \leq\left(u_{1}-1\right) t \\
v_{1}, x>\left(u_{1}-1\right) t
\end{array}\right.
\end{aligned}
$$


(1-rarefaction wave), where $C_{1}=v_{0}-u_{0}^{2} / 2-u_{0}-1 / 2$, and $\left(u_{1}, v_{1}\right)$ lies in a 1 -rarefaction curve starting at the point $\left(u_{0}, v_{0}\right)$. Or

$$
\begin{gathered}
u(x, t)=\left\{\begin{array}{c}
u_{0}, x<\left(u_{0}+1\right) t \\
x / t-1,\left(u_{0}+1\right) t \leq x \leq\left(u_{1}+1\right) t \\
u_{1}, x>\left(u_{1}+1\right) t
\end{array}\right. \\
v(x, t)=\left\{\begin{array}{c}
v_{0}, x<\left(u_{0}+1\right) t \\
(x / t)^{2} / 2-2 x / t+C_{2},\left(u_{0}+1\right) t \leq x \leq\left(u_{1}+1\right) t \\
v_{1}, x>\left(u_{1}+1\right) t
\end{array}\right.
\end{gathered}
$$

(2-rarefaction wave), where $C_{2}=v_{0}-u_{0}^{2} / 2+u_{0}-1 / 2$, and $\left(u_{1}, v_{1}\right)$ lies in a 2 -rarefaction curve starting at the point $\left(u_{0}, v_{0}\right)$.

(c) Singular shock waves (see Definition 2.8) of 3'SD-type:

$$
\begin{aligned}
& u(x, t)=\left\{\begin{array}{l}
u_{0}, x<c t \\
u_{1}, x>c t
\end{array}\right\}+\tilde{s}(t)\left(\alpha_{0} d_{\varepsilon}^{-}(x-c t)+\alpha_{1} d_{\varepsilon}^{+}(x-c t)\right) \\
& v(x, t)=\left\{\begin{array}{l}
v_{0}, x<c t \\
v_{1}, x>c t
\end{array}\right\}+s(t)\left(\beta_{0} D_{\varepsilon}^{-}(x-c t)+\beta_{1} D_{\varepsilon}^{+}(x-c t)\right),
\end{aligned}
$$

where $c=\left[u^{2}-v\right] /[u]$, and all other terms are as in that definition. That means

$$
D_{\varepsilon} \approx \delta,\left(d_{\varepsilon}^{ \pm}\right)^{i} \approx 0, i=1,3,\left(d_{\varepsilon}^{ \pm}\right)^{2} \approx \delta
$$

while $\left(u_{1}, v_{1}\right)$ lies in a region denoted by $Q_{7}$ in [6] of the point $\left(u_{0}, v_{0}\right)$ (see Figure 4.1).

For an arbitrary Riemann problem to (1.2), one can construct a solution by the means of these waves or their combinations ([6]).

While interactions of the first two types can be handled in the usual ways, interactions involving DSSW are quite different and far more interesting, so they become a topic of this paper.

The procedure for DSSW interactions can also be used for systems (1.1), but a complete after-interaction solution depends highly on a particular system. That is the reason why we treat system (1.2) only.

In order to simplify notation, we shall substitute the point $(X, T)$ in (3.3) by $(0,0)$ and then solve the Cauchy problem (1.2, 3.3).

There is no multiplication of $v$ with $u$ in system (1.2), so in the sequel it will suffice to take $D^{-}=D^{+}, \alpha_{0}(t):=\alpha_{0} \tilde{s}(t), \alpha_{1}(t):=\alpha_{1} \tilde{s}(t)$, and $\beta(t):=s(t)$, i.e. to look for a solution of the form

$$
\begin{aligned}
& u=G(x-c t)+\left(\alpha_{0}(t) d^{-}(x-c t)+\alpha_{1}(t) d^{+}(x-c t)\right) \\
& v=H(x-c t)+\beta(t) D(x-c t),
\end{aligned}
$$

where $G$ and $H$ are generalized step functions, $d$ is a $3^{\prime} \mathrm{SD}$-function, $D$ is $\mathrm{S} \delta$-function, and $c \in \mathbb{R}$.

Let us determine SDSL of (1.2) for some $\left(u_{0}, v_{0}\right) \in \mathbb{R}^{2}$. 
Substitution of (4.6) into the first equation of the system gives

$$
\begin{aligned}
& c=\frac{u_{1}^{2}-v_{1}-u_{0}^{2}+v_{0}}{u_{1}-u_{0}} \\
& \alpha_{0}^{2}(t)+\alpha_{1}^{2}(t)=\beta(t),
\end{aligned}
$$

where $c$ is the speed of the wave. After neglecting all terms converging to zero as $\varepsilon \rightarrow 0$, the second equation becomes

$$
\begin{aligned}
& \partial_{t} H_{\varepsilon}(x-c t)+\beta^{\prime}(t) \delta(x-c t)-c \beta(t) \delta^{\prime}(x-c t)+\partial_{x}\left(\frac{1}{3} G_{\varepsilon}^{3}-G\right) \\
& +\left(u_{1} \alpha_{0}^{2}(t)+u_{0} \alpha_{1}^{2}(t)\right) \delta^{\prime}(x-c t)=0 .
\end{aligned}
$$

Thus, the following relations have to hold.

$$
\beta^{\prime}(t)=c\left(v_{1}-v_{0}\right)-\left(\frac{1}{3} u_{0}^{3}-u_{0}-\frac{1}{3} u_{1}^{3}+u_{1}\right)=: k,
$$

i.e.

$$
\beta(t)=k t+\zeta, \text { since } \beta(0)=\zeta
$$

and

$$
u_{1} \alpha_{0}^{2}(t)+u_{0} \alpha_{1}^{2}(t)=c \beta(t) .
$$

Like in [6], one can see that the overcompressiveness means

$$
u_{0}-1 \geq c \geq u_{1}+1
$$

i.e., $v_{1}$ lies between the curves

$$
\begin{aligned}
& D=\left\{(u, v): v=v_{0}+u^{2}+u-u_{0} u-u_{0}\right\} \\
& E=\left\{(u, v): v=v_{0}-u+u_{0} u-u_{0}^{2}+u_{0}\right\},
\end{aligned}
$$

and $u_{0}-u_{1} \geq 2$.

Denote by $J_{1}$ the union of the parts of the admissible Hugoniot locus:

$$
S_{1}=\left\{(u, v): v-v_{0}=\left(u-u_{0}\right)\left(\frac{u_{0}+u}{2}+\sqrt{1-\frac{\left(u_{0}-u\right)^{2}}{12}}\right)\right\},
$$

and

$$
S_{2}=\left\{\left(u_{1}, v_{1}\right): v-v_{0}=\left(u-u_{0}\right)\left(\frac{u_{0}+u}{2}-\sqrt{1-\frac{\left(u_{0}-u\right)^{2}}{12}}\right)\right\},
$$

for $u \in\left[u_{0}-\sqrt{12}, u_{0}-3\right]$. Note that $S_{i}$ is not an $i$ th shock curve but only a label.

The points between the curves $D$ and $E$, and on the left-hand side of $J_{1}$, define the area denoted by $Q_{7}$ in [6]. Here, this area is called the delta singular locus.

One can easily check that system (4.7, 4.9) has a solution if and only if $\beta(t)>0$. Depending on $k$, defined in (4.8), there are three possibilities for a resulting wave:

(i) If $k>0$, then $\tilde{\beta}^{\prime}(t)>0$ and $\left(u_{1}, v_{1}\right) \in Q_{7}$. The resulting DSSW has the same properties as before, i.e. its strength increases with the time.

(ii) If $k=0$, then $\tilde{\beta} \equiv$ const $=\zeta>0$ and the corresponding part of a singular overcompressive locus is $J_{1}$. The result of the interaction is a new kind of DSSW, and its strength is a constant with respect to the time.

(iii) If $k<0$ (this means that the point $\left(u_{1}, v_{1}\right)$ is on the left-hand side of $J_{1}$ ), then the resulting DSSW is quite different than the usual one (with an increasing strength). 


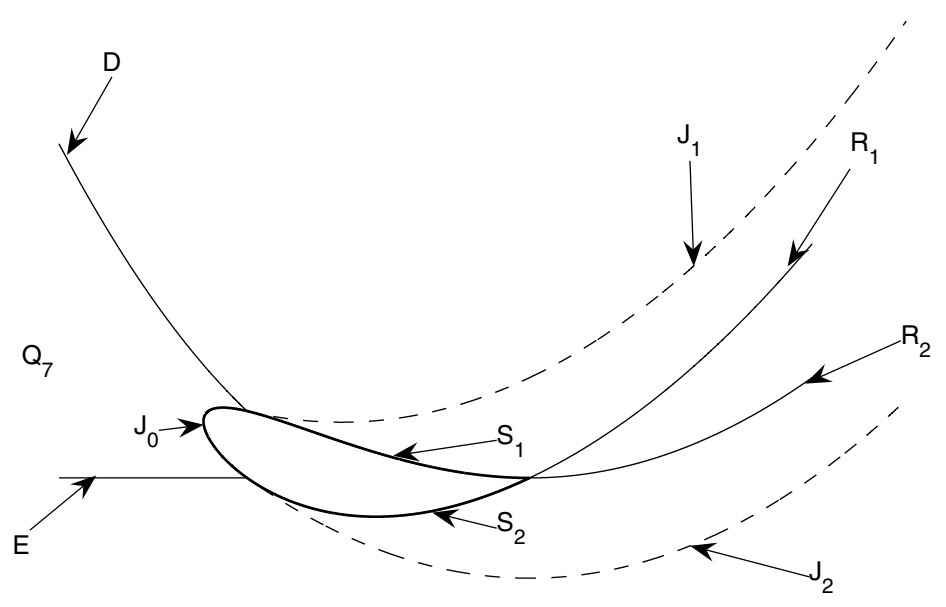

FIG. 4.1

Its initial strength equals $\zeta, \beta(0)=\zeta>0$, but linearly decreases in time. At some point $T_{0}$ the strength of the DSSW equals zero and the singular shock wave does not exist after that. In the remainder of the paper we shall study some cases when this happens. The new initial data for time $t=T_{0}$ are the Riemann ones, and the solution after that time can be found in the usual way, by using the results in [6].

All of the above facts are collected in the following theorem.

Theorem 4.1. The $\mathrm{SDSL}_{\zeta}, \zeta>0$, for (1.2, 3.3) is the area bounded by the curves $D$, $E, S_{2} \backslash J_{0}$, and $S_{1} \backslash J_{0}$. (The area $Q_{7}$ is a subset of this one, as known from Lemma 3.2.) The overcompressive $\operatorname{SDSL}_{\zeta}, \zeta>0$, is a part of the SDSL bounded by the curves $D$ and $E$ such that $u_{1} \leq u_{0}-2$.

4.1. Interaction of a DSSW and an admissible shock wave. Suppose that a DSSW with a speed $c_{1}$ and left- and right-hand values $U_{0}=\left(u_{0}, v_{0}\right)$ and $U_{1}=\left(u_{1}, v_{1}\right)$, respectively, interact with an admissible shock wave with a speed $c_{2}<c_{1}$ having left-hand and righthand values $U_{1}=\left(u_{1}, v_{1}\right)$ and $U_{2}=\left(u_{2}, v_{2}\right)$, respectively, at a point $(X, T)$.

LEMmA 4.2. If the above DSSW and shock wave are admissible, then $\left(u_{2}, v_{2}\right)$ lies between the lines $D$ and $E$. Thus, the solution after the interaction is a single overcompressive DSSW.

Proof. Since $u_{0} \geq u_{1}+3$ and $u_{1}>u_{2}$ (because of the admissibility conditions for singular and shock waves), we have $u_{0}>u_{2}+3$. The point $\left(u_{2}, v_{2}\right)$ lies on the curve $S_{1}$ 
or $S_{2}$ with the origin at the point $\left(u_{1}, v_{1}\right)$. Thus

$$
v_{2}=v_{1}+\left(u_{2}-u_{1}\right)\left(\frac{u_{1}+u_{2}}{2} \pm \sqrt{1-\frac{\left(u_{1}-u_{2}\right)^{2}}{12}}\right) .
$$

The point $\left(u_{1}, v_{1}\right)$ lies in the area denoted by $Q_{7}$, and thus below or at the curve $D$ with the origin at $\left(u_{0}, v_{0}\right)$. Therefore

$$
v_{1} \leq v_{0}+u_{1}^{2}+u_{1}-u_{0} u_{1}-u_{0} .
$$

Let the point $\left(u_{0}, v_{0}\right)$ be the origin. The point $\left(u_{2}, v_{2}\right)$ will be below the curve $D$ if

$$
\begin{aligned}
& v_{0}+u_{1}^{2}+u_{1}-u_{0} u_{1}-u_{0}+\left(u_{2}-u_{1}\right)\left(\frac{u_{1}+u_{2}}{2} \pm \sqrt{1-\frac{\left(u_{1}-u_{2}\right)^{2}}{12}}\right) \\
\leq & v_{0}+u_{2}^{2}+u_{2}-u_{0} u_{2}-u_{0} .
\end{aligned}
$$

Non-positivity of $u_{1}-u_{2}$ gives

$$
\pm \sqrt{1-\frac{\left(u_{1}-u_{2}\right)^{2}}{12}} \leq \frac{1}{2}\left(u_{0}-u_{1}\right)+\frac{1}{2}\left(u_{0}-u_{2}\right)-1 .
$$

The left-hand side of the above inequality is less than 2 , while the right-hand side is greater than 2 . Thus, the point $\left(u_{2}, v_{2}\right)$ really lies below the curve $D$.

In the same way one can prove that the point $\left(u_{2}, v_{2}\right)$ lies above the curve $E$.

REMARK 4.3. In the same manner as above, one can prove that the situation is the same when a DSSW and a shock wave change sides. That is, when an admissible DSSW interacts with an admissible shock wave from the right-hand side, then the solution is again a single admissible DSSW.

4.2. Double DSSW wave interaction. Suppose that an admissible DSSW with a speed $c_{1}$ and left- and right-hand side values $U_{0}=\left(u_{0}, v_{0}\right)$ and $U_{1}=\left(u_{1}, v_{1}\right)$, respectively, interacts with another DSSW with a speed $c_{2}<c_{1}$ and left-hand (right-hand) side values $U_{1}=\left(u_{1}, v_{1}\right)\left(U_{2}=\left(u_{2}, v_{2}\right)\right)$ at the point $(X, T)$. Since the conditions for the existence of a DSSW include $u_{0}-u_{1} \geq 3$ and $u_{1}-u_{2} \geq 3$, then $u_{0}-u_{2} \geq 6$, i.e. the point $\left(u_{2}, v_{2}\right)$ is on the left-hand side of the line $u=u_{0}-\sqrt{12}$. Concerning the position of the point $\left(u_{2}, v_{2}\right)$ in the plane of wave regions with the origin at $\left(u_{0}, v_{0}\right)$, there are three possibilities:

(i) The point $\left(u_{2}, v_{2}\right)$ is between or at the curves $D$ and $E$. The result of the interaction is a single DSSW (with increasing strength).

(ii) The point $\left(u_{2}, v_{2}\right)$ is above the curve $D$. The result of the interaction is a 1rarefaction wave followed by a DSSW.

(iii) The point $\left(u_{2}, v_{2}\right)$ is below the curve $E$. The result of the interaction is a DSSW followed by a 2-rarefaction wave.

SDSL's always have increasing strength in these three cases.

5. Interaction of a DSSW and a rarefaction wave. The last possibility of DSSW interaction is with a rarefaction wave. That possibility is omitted from considerations of the general system (1.1) due to a richness of possible behaviors. Nevertheless, the majority of specific Riemann problems can be treated similarly as system (1.2) was here, at least up to some point. 
For a given point $\left(u_{0}, v_{0}\right)$, the rarefaction curves are given by (see [6])

$$
\begin{aligned}
& R_{1}=\left\{(u, v): v=v_{0}-\frac{1}{2} u_{0}^{2}+\frac{1}{2} u^{2}+u-u_{0}\right\}, \\
& R_{2}=\left\{(u, v): v=v_{0}-\frac{1}{2} u_{0}^{2}+\frac{1}{2} u^{2}-u+u_{0}\right\} .
\end{aligned}
$$

Suppose that a DSSW coming from the left-hand side with left- and right-hand side values $U_{0}=\left(u_{0}, v_{0}\right)$ and $U_{1}=\left(u_{1}, v_{1}\right)$ interacts with a rarefaction wave at some point $(X, T)$. If the rarefaction wave is approximated with a number of small-amplitude (nonadmissible) shock waves like in the wave front tracking algorithm (see [1] for example), intuition given in Theorem 3.5 indicates that the first task should be to look at the DSSW and the interaction of the DSSW and a non-admissible shock wave. It is possible to extend Theorem 3.5 for such a case, providing that a non-admissible shock wave has amplitude small enough (of the rate $\varepsilon^{2}$, say). Denote by $\left(u_{r}, v_{r}\right)$ the end-point in a rarefaction curve. Let us note that the starting point of the curve $\left(u_{1}, v_{1}\right)$ is in $Q_{7}$.

In what follows, we shall abuse the notation and denote by $\left(u_{1}, v_{1}\right) \in Q_{7}$ the left-hand side and by $\left(u_{2}, v_{2}\right)$ the right-hand side value of a part from the rarefaction curve. These values will represent constant states in an approximated non-admissible shock wave, too. If $\left(u_{2}, v_{2}\right) \in Q_{7}$, then the result of the interaction is a single DSSW with the left-hand side values equal to $\left(u_{0}, v_{0}\right)$. The speed depends on initial values as in (4.7). So, one can continue the procedure taking approximate points from the rarefaction curve as the right-hand values of the non-admissible shock wave until it reaches the border of $Q_{7}$.

After looking at the above discrete model we return to a real situation.

Let us denote by $(c(t), t), t$ belonging to some interval, a path of the resulting DSSW through $Q_{7}$. It is possible to explicitly calculate the above path. For example, if a DSSW interacts with a centered 1-rarefaction wave, by substituting

$$
\begin{aligned}
& u(x, t)=\left\{\begin{array}{r}
u_{1}, x<c(t) \\
\phi_{1}(x / t), x>c(t)
\end{array}\right\}+\alpha_{0}(t) d^{-}(x-c(t))+\alpha_{1}(t) d^{+}(x-c(t)) \\
& v(x, t)=\left\{\begin{array}{r}
v_{1}, x<c(t) \\
\phi_{2}(x / t), x>c(t)
\end{array}\right\}+\beta(t) D(x-c(t))
\end{aligned}
$$

in system (1.2), one obtains

$$
\begin{aligned}
& {\tilde{\alpha_{0}}}^{2}(t)+\tilde{\alpha}_{1}^{2}(t)=\tilde{\beta}(t) \\
& c(t)=\left(t\left(1-2\left(u_{1}-v_{0}+v_{1}+u_{0}^{2}-u_{1}^{2}\right)\right)\right. \\
& \left.+T\left(1-2\left(u_{0}-v_{1}-u_{0} u_{1}+u_{0}^{2}-u_{1}^{2}\right)\right)\right) /\left(2\left(u_{0}-1\right)\right) \\
& \tilde{\beta}^{\prime}(t)=c^{\prime}(t)\left(\frac{1}{2}\left(\frac{c(t)}{t}+1\right)+\left(\frac{c(t)}{t}+1\right)+v_{1}-\frac{1}{2} u_{1}^{2}-u_{1}-v_{0}\right) \\
& -\left(\frac{1}{3}\left(\frac{c(t)}{t}+1\right)^{3}-\left(\frac{c(t)}{t}+1\right)-\frac{1}{3} u_{0}^{3}+u_{0}\right),
\end{aligned}
$$

where the initial data for $\beta$ at the point $t=T$ is the initial strength of the DSSW $\beta(T)$. The above calculations means that a form of the resulting singular shock curve and its strength are uniquely determined through the area $Q_{7}$. If $\left(u_{r}, v_{r}\right) \in Q_{7}$, then the 
analysis is finished. Suppose that this is not true. The main problem then is to analyze the situation when a rarefaction curve intersects the boundary of $Q_{7}$. Let us try to find out what is happening by using a discrete model.

The first real problem is to find a form of solution when the points from the rarefaction curve satisfy $\left(u_{1}, v_{1}\right) \in Q_{7}$ and $\left(u_{2}, v_{2}\right) \notin Q_{7}$.

Denote by $\tilde{D}$ and $\tilde{G}$ the intersection points of the curve $J_{1}$ (or the line $u=u_{0}-3$ ) with the curves $E$ and $D$, respectively (see Figure 5.1).

5.1. The first critical case. Denote by $J$ the 1-rarefaction curve starting from the point $\tilde{G}$ and by $J_{2}$ the 2-rarefaction curve starting from the point $\tilde{D}$. The region where $\left(u_{2}, v_{2}\right)$ can lie consists of five subregions:

(i) The rarefaction curve which starts at $\left(u_{1}, v_{1}\right)$ intersects the curve $D$ out of point $\tilde{G}$. The point $\left(u_{2}, v_{2}\right)$ lies in the region above the curve $D$ and left of the line $u=u_{0}-3$. The final result of the interaction is a 1-rarefaction wave $\left(R_{1}\right)$ followed by a DSW with increasing strength.

(ii) The rarefaction curve which starts at $\left(u_{1}, v_{1}\right)$ intersects the curve $E$ out of point $\tilde{D}$. The point $\left(u_{2}, v_{2}\right)$ lies in the region below the curve $E$ and on the left-hand side of the line $u=u_{0}-3$. The result of the interaction is a DSSW with increasing strength followed by a 2 -rarefaction wave $\left(R_{2}\right)$.

(iii) The rarefaction curve which starts at $\left(u_{1}, v_{1}\right)$ intersects the curve $J_{1}$ out of points $\tilde{D}$ and $\tilde{G}$. Since an amplitude of a non-admissible shock wave can be as small as necessary, one can assume that the point $\left(u_{2}, v_{2}\right)$ lies in the second delta singular locus and the resulting DSSW has a negative strength. The strength-function $\tilde{\beta}(t)=\zeta+k\left(t-T_{0}\right)$ of the resulting DSSW is decreasing, so there could exist a point $T_{1}=T-\zeta / k$ such that $\tilde{\beta}\left(T_{1}\right)=\alpha_{0}=\alpha_{1}=0$. Let $X_{1}=c T_{1}+(X-T)$, where $c$ is the speed of the resulting DSSW (space coordinate of the point where strength reaches zero). Therefore, in the time $t=T_{1}$, we have to solve a new Riemann problem:

$$
\left.u\right|_{t=T_{1}}=\left\{\begin{array}{ll}
u_{0}, & x<X_{1} \\
u_{2}, & x>X_{1}
\end{array},\left.v\right|_{t=T_{1}}=\left\{\begin{array}{ll}
v_{0}, & x<X_{1} \\
v_{2}, & x>X_{1}
\end{array} .\right.\right.
$$

This problem has a unique entropy solution that consists of two shock waves, since the point $\left(u_{2}, v_{2}\right)$ is between the curves $S_{1}$ and $S_{2}$, with respect to the origin at the point $\left(u_{0}, v_{0}\right)$. This means that the singular shock wave decouples into a pair of admissible shock waves. If the decoupling starts before $u$ reaches the value $\min \left\{u_{r}, u_{0}-2\right\}$, then the result of the interaction will be known after solving shock - rarefaction wave interactions. In any case there are no DSSWs in the solution after time $t=T_{1}$. If $u_{r} \leq u_{0}-2$ and the decoupling does not start before $u$ reaches the value $u_{r}$, then this pair of shock waves is the final solution. The case when $u_{r}>u_{0}-2$ belongs to the following subsection, i.e. the second critical case.

(iv) The rarefaction curve $R_{j}, j=1$ or 2 , which starts at $\left(u_{1}, v_{1}\right)$ intersects the curve $J_{1}$ in the point $\tilde{G}$. We can take $\tilde{G}=\left(u_{2}, v_{2}\right)$ for convenience. The set of such points 
$\left(u_{1}, v_{1}\right)$ lies on the inverse rarefaction curve (it starts from the right-hand side values),

$$
\begin{aligned}
& \tilde{R}_{1}=\left\{(u, v): v=v_{0}+\left(u_{0}^{2}-u^{2}\right) / 2+u_{0}-u\right\} \\
& \quad \text { and } \\
& \tilde{R}_{2}=\left\{(u, v): v=v_{0}+\left(u_{0}^{2}-u^{2}\right) / 2-u_{0}+u\right\} .
\end{aligned}
$$

A straightforward calculation shows that this curve lies in the region $Q_{7}$; thus, this situation is possible, as one can see using the inverse rarefaction curves $\tilde{R}_{1}$ and $\tilde{R}_{2}$ given above.

If $j=1$, then the point $\left(u_{2}, v_{2}\right)$ belongs to $J$ and the solution after the interaction is an $R_{1}$-wave followed by a DSSW with a constant strength.

If $j=2$, then the point $\left(u_{2}, v_{2}\right)$ lies in the area below the curve $J$. This can be verified by direct calculation, taking into account that the amplitude of a non-admissible shock is small enough, $u_{2}<u_{0}-2$. The solution after the interaction is an admissible DSSW with a decreasing strength. Further explanations of such a DSSW are given in the following subsection.

(v) The rarefaction curve $R_{j}$ which starts at $\left(u_{1}, v_{1}\right)$ intersects the curve $J_{1}$ in the point $\tilde{D}$. Again, let $\tilde{D}=\left(u_{2}, v_{2}\right)$. Simple calculation, as in case (iv), shows that this situation is also possible since the inverse rarefaction curves starting from $\tilde{D}$ stay in $Q_{7}$. If $j=2$, the point $\left(u_{2}, v_{2}\right)$ belongs to $J_{2}$, and then the solution after the interaction is a DSSW with a constant strength followed by an $R_{2}$-wave.

If $j=1$, then use of the same arguments as above yields that the point $\left(u_{2}, v_{2}\right)$ lies in the area above the curve $J_{2}$ and the result of the interaction is an admissible DSSW with a decreasing strength. Again, one can see the following subsection for further analysis.

5.2. The second critical case. Now we are dealing with the problem when the rarefaction wave passes through the curves $D$ or $E$, after passing through $J_{1}$. One can see that this is the continuation of the cases (iii)-(v) above.

(a) Denote by $\hat{D}$ the area above the curve $D$, below $S_{1}$ and on the left-hand side of the line $u=u_{0}-2$. Also denote by $\hat{\hat{D}}$ the area above the curve $E$, below $S_{1}$ and on the right-hand side of the line $u=u_{0}-2$. If $\left(u_{2}, v_{2}\right)$ lies in one of these regions, then the solution is a combination of a rarefaction wave $R_{1}$ and an overcompressive DSSW with a decreasing or constant strength.

(b) Denote by $\hat{E}$ the area below the curve $E$, above $S_{2}$ and on the left-hand side of the line $u=u_{0}-2$. Also denote by $\hat{\hat{E}}$ the area below the curve $D$, above $S_{2}$ and on the right-hand side of the line $u=u_{0}-2$. If $\left(u_{2}, v_{2}\right)$ lies in one of these regions, the solution is then a combination of an overcompressive DSSW with a decreasing or constant strength and a rarefaction wave $R_{2}$. Denote by $D_{0}$ the area bounded by the curves $D, E, S_{1}$, and $S_{2}$ such that $u<u_{0}-2$ in $D_{0}$.

One can see that a rarefaction curve cannot enter into $D_{0}$ since it has to pass through the intersection point $\left(u_{0}-2,-2 u_{0}+v_{0}+2\right)$ of $D$ and $E$, but

$$
\begin{aligned}
& 2 u-2 u_{0}-u u_{0}+u^{2} / 2+u_{0}^{2} / 2+2>0 \text { (i.e. } R_{1} \text { is above the curve } E \text { ) } \\
& 2 u_{0}-2 u+u u_{0}-u^{2} / 2-u_{0}^{2} / 2-2<0 \text { (i.e. } R_{2} \text { is below the curve } D \text { ). }
\end{aligned}
$$




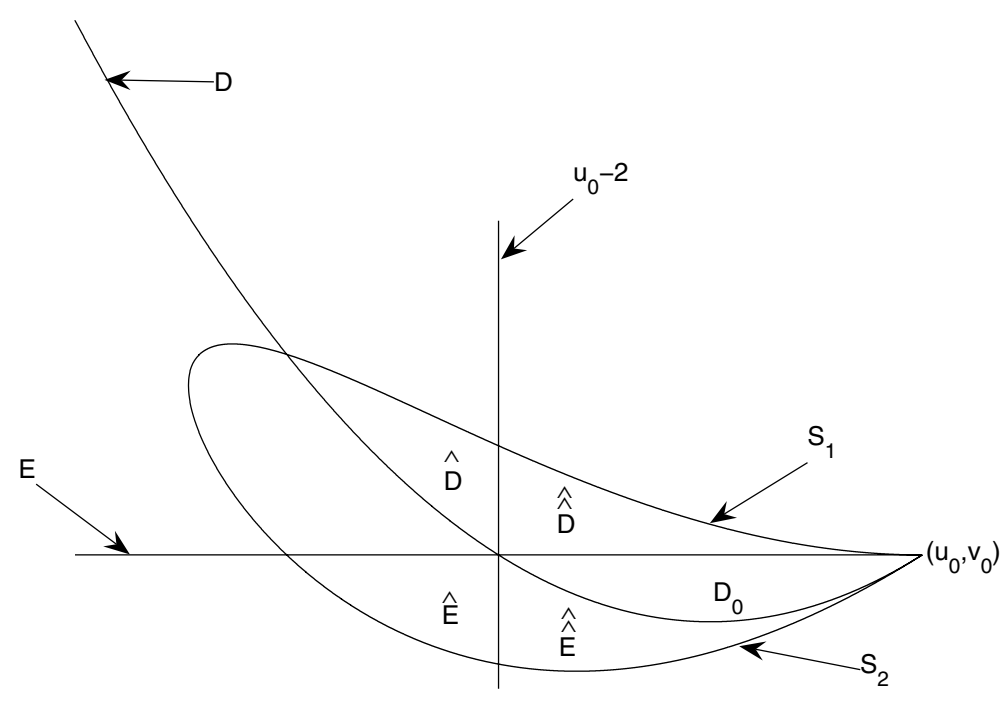

FIG. 5.1

Therefore, a rarefaction curve which passes through the point $D \cap E$ goes either into $\hat{\hat{D}}$ or $\hat{\hat{E}}$, and these cases are analyzed above.

Thus, we have described all important points of the interactions between DSSWs and rarefaction waves. When a result of a single interaction is known, the question about further singular shock path could be answered by a successive use of the above procedures.

REMARK 5.1. One can use similar analysis of all possible cases when a rarefaction wave which interacts with a DSSW is on the left-hand side of it. Instead of direct rarefaction and singular shock curves, the inverse ones should be used; i.e. $\left(u_{2}, v_{2}\right)$ is a starting point and one is able to calculate $v_{0}$ from formulas of $E, D, S_{1}, S_{2}, R_{1}$, and $R_{2}$.

REMARK 5.2. In contrast to the case in [9, where interaction can generate some "strange" solution containing an unbounded $L_{l o c}^{1}$ function, in the present system one can find only bounded functions and DSSWs as a result on an interaction.

For a system (1.1) with $g_{1} \not \equiv$ const, or $g_{2} \not \equiv 0$, the interaction of DSSWs and rarefaction waves cannot be treated as easily as above.

Thus, we have proved the following assertion for the interaction in the case of system (1.2).

TheOREm 5.3. Suppose that a DSSW interacts with a rarefaction wave at the time $T$. For some time period $T<t<T_{1}$, the solution is represented by a DSSW wave supported by a uniquely defined curve (not a line) followed by a new rarefaction wave. Depending on the right-hand value of the primary rarefaction wave, one has the following possible cases for a solution after $t>T_{1}$.

(a) Single DSSW (supported by a line) with an increasing strength. 
(b) 1-rarefaction wave followed by a DSSW with an increasing strength.

(c) Singular shock wave with an increasing strength followed by a 2-rarefaction wave.

(d) Singular shock wave with a decreasing strength prolonged by either a single DSSW with an increasing strength, or a pair of admissible shock waves.

(e) 1-rarefaction wave followed by a DSSW with a constant strength.

(f) Singular shock wave with a constant strength followed by a 2-rarefaction wave.

(g) Singular shock wave with a decreasing strength prolonged by either a 1-rarefaction wave followed by a DSSW with decreasing or constant strength, or a DSSW with decreasing or constant strength followed by a 2-rarefaction wave.

"Prolonged" is the state after the strength of a DSSW becomes zero. Such a wave can also stop with non-zero strength, and then there is obviously no prolongation as described above.

\section{REFERENCES}

[1] A. Bressan, Hyperbolic Systems of Conservation Laws, Oxford University Press, New York, 2000. MR 1816648 (2002d:35002)

[2] C. Dafermos, Hyperbolic Conservation Laws in Continuum Physics, Springer-Verlag, Heidelberg, 2000. MR1763936 (2001m:35212)

[3] V. G. Danilov and V. M. Shelkovich, Dynamics of propagation and interaction of shock waves in conservation law systems, J. Differ. Equations 211 (2005), 333-381. MR2125546 (2006f:35173)

[4] _ Delta-shock wave type solution of hyperbolic systems of conservation laws, Q. Appl. Math. 29 (2005), 401-427. MR2169026 (2006j:35158)

[5] F. Huang, Weak solution to pressureless type system, Comm. Partial Differential Equations 30 (2005), no. 1-3, 283-304. MR2131055 (2005k:35263)

[6] B. L. Keyfitz and H. C. Kranzer, Spaces of weighted measures for conservation laws with singular shock solutions, J. Differ. Equations 118 (1995), no. 2, 420-451. MR1330835 (96b:35138)

[7] P. D. Lax, Hyperbolic Systems of Conservation Laws and the Mathematical Theory of Shock Waves, SIAM, Philadelphia, 1973. MR0350216 (50:2709)

[8] M. Nedeljkov, Delta and singular delta locus for one dimensional systems of conservation laws, Math. Method Appl. Sci. 27 (2004), 931-955. MR2055283 (2005g:35210)

[9] M. Nedeljkov and M. Oberguggenberger, Delta shock wave and interactions in a simple model case, Submitted.

[10] Tan, D., Zhang, T. and Zheng, Y., Delta-shock waves as limits of vanishing viscosity for hyperbolic systems of conservation laws, J. Differ. Equations 112 (1994), 1-32. MR1287550 (95g:35124) 